boundary with a S-value of $5 \mathrm{~S}$ may be explained as being due to soluble protein and RNA.

Plate l(b), (c) and (d) were the patterns obtained with E. coli extract irradiated with X-rays to 60,100 and 140 krads respectively. Plate $1(\mathrm{e})$ shows the pattern when the extract was irradiated to $147 \mathrm{krads}$ with $\beta$-rays. The general nature of the patterns obtained with extracts after irradiation was similar to that obtained in the control pattern. However, one interesting point to note is that in the pattern obtained with unirradiated cell extract the ratio of the $85 \mathrm{~S}$ to the $70 \mathrm{~S}$ boundaries, as judged from the area of the peaks, was close to unity. In a series of experiments, it was observed that with increasing dose, the amount of the $85 \mathrm{~S}$ component gradually decreased with a consequent increase in the amount of the $70 \mathrm{~S}$ peak.
It has been suggested that the $70 \mathrm{~S}$ particle in bacteria represent an inactive form of ribosome possessing the capacity for protein synthesis. When it actively synthesises protein it sediments with a higher value of $85 \mathrm{~S}$. The results of this study also suggest a possible relationship between the $70 \mathrm{~S}$ and the $85 \mathrm{~S}$ ribosomes. The sensitivity to ionising radiation of all the ribosomes are not similar, the $85 \mathrm{~S}$ component being most sensitive in this respect. The decrease in the amount of the $85 \mathrm{~S}$ component with a consequent increase in the $70 \mathrm{~S}$ fraction indicates a possible relationship of the type $85 \mathrm{~S} \rightleftarrows 70 \mathrm{~S}+\mathrm{X}$. X may represent nascent protein and RNA molecules.

This work was carried out under the financial sponsorship of the Ministry of Scientific Research and Cultural Affairs, Govt. of India.

\section{Gewinnung und Eigenschaften der Neuro- hormone aus den Augenstielen von Leander adspersus}

\author{
Werner Kapitza * und Friedrich Fischer \\ Institut für Pflanzenchemie der Technischen Universität \\ Dresden, Tharandt
}

(Z. Naturforschg. 21 b, 712-713 [1966] ; eingegangen am 25. April 1966)

Die aus den schon beschriebenen Untersuchungen erkannte Gleichheit der Wirkstoffe bei Insekten und Crustaceen ergab für die Isolierung der Neurohormone eine günstige Materialquelle in den Augenstielen der Garnelen ${ }^{1-6}$. Die Aufarbeitung der Augenstiele der Garnele Leander adspersus mit den bekannten Methoden der Vorextraktion und Papierchromatographie ${ }^{7}$ führte jedoch nur zu sehr unreinen Produkten; die Neurohormone wurden im Gemisch mit Pterinen und Aminosäuren isoliert ${ }^{1,4,5}$. Da mit den chromatographischen Methoden jedoch eine gute Auftrennung der beiden biologisch aktiven Substanzen gelang, wurde zunächst eine säulenchromatographische Trennung des Wasserextraktes von Leander adspersus an CellulosePulver mit dem Lösungsmittelgemisch n-Propanol/Ammoniak durchgeführt, um dann im 2. Schritt die Neurohormone von den Aminosäuren zu trennen.

Der Verlauf der Fraktionierung wurde durch Nachweisreaktionen für Aminosäuren und mit Hilfe der biologischen Testung verfolgt. Die Fraktionen des Neurohormones $\mathrm{C}$ zeigten nach der papierchromatographi-

* Teil aus der Dissertation von W. Kapitza, TU Dresden, 1965, weitere Teile dieser Dissertation s. l. c. ${ }^{1-6}$.

1 F. Fischer, W. Kapitza, M. Gersch u. H. Unger, Z. Naturforschg. 17 b, 834 [1962].

2 M. Gersch, H. Unger, F. Fischer u. W. Kapitza, Z. Naturforschg. 18 b, 587 [1963].

3 M. Gersch, H. Unger, F. Fischer u. W. Kapitza, Zool. Jb. Physiol. 70, 455 [1964]. schen Kontrolle in n-Butanol/Äthanol/Eisessig/Wasser $(8: 2: 1: 3)$ (BÄEW) jeweils nur einen mit Serin und Glycin lagegleichen Fleck mit der Aktivität des Neurohormones C, während die Fraktionen des Neurohormones $\mathrm{D}$ in den mit Threonin und Alanin lagegleichen Bereich mit der Wirksamkeit des Neurohormones D und inaktives Taurin ausgetrennt wurden.

Die hochaktiven Eluate der Neurohormone C und D aus den B ÄEW-Chromatogrammen wurden mit 2.4-Dinitrofluorbenzol in die DNP-Derivate umgewandelt und diese Dünnschicht-chromatographisch auf Kieselgel G mit unter den gleichen Bedingungen hergestellten DNP. Aminosäuren verglichen. Es ließen sich neben 2.4-Dinitrophenol und 2.4-Dinitroanilin jeweils zwei verschiedene DNP-Derivate nachweisen, die mit den DNP. Aminosäuren von Alanin und Threonin (Eluat Neurohormon D) bzw. Glycin und Serin (Eluat Neurohormon C) nach der Chromatographie in verschiedenen Lösungsmittelgemischen ein identisches Verhalten zeigten. DNP-Derivate der Neurohormone C und D konnten dabei nicht abgetrennt werden.

Da kaum zu erwarten war, da $\beta$ auch die DNP-Derivate der Neurohormone mit den entsprechenden DNP. Aminosäuren ein identisches chromatographisches Verhalten besitzen, wurde angenommen, daß die Neurohormone in Mengen unterhalb der chemisch erfaßbaren Nachweisgrenze im Arthropodenorganismus vorkommen. Das würde dem bekannten Ergebnis der $2 \cdot 10^{7}$. fachen Anreicherung bei der Isolierung des Insektenhormones Ecdyson aus Seidenspinner-Puppen entsprechen ${ }^{8}$.

4 F. Fischer, W. Kapitza, M. Gersch u. H. Unger, Beiträge zur Biochemie und Physiologie von Naturstoffen, Festschrift Kurt Mothes zum 65. Geburtstag, Jena 1965, S. 121.

5 W. Kapitza, Mitt. Bl. Chem. Ges. DDR 12, 276 [1965].

${ }^{6}$ F. Fischer u. W. Kapitza, Z. Naturforschg. 20 b, 1311 [1965].

7 M. Gersch, F. Fischer, H. Unger u. H. Кoch, Z. Naturforschg. 15 b, 319 [1960].

8 A. Butenandt u. P. Karlson, Z. Naturforschg. 9 b, 389 [1954]. 
Bestätigt wurde die Annahme der für einen chemischen Nachweis zu geringen Konzentrationen der Neurohormone durch die Reinigung der säulenchromatographisch getrennten Fraktionen der Neurohormone $\mathrm{C}$ und D an Sephadex G-25 mit 0,05-n. Ammoniak (Abb. 1). Durch diese Operation gelang es erstmalig, die Neurohormone $\mathrm{C}$ und $\mathrm{D}$ in reiner Form zu isolieren. Die biologische Aktivität dieser mit chemischen Methoden nicht nachweisbaren Mikrosubstanzen wurde im PeriplanetaHerztest nach Unger ${ }^{9}$, im elektrophysiologischen Test nach UNGER und KALKoFF ${ }^{10}$ und im Farbwechseltest an den Garnelen Leander adspersus und Crangon vulgaris ${ }^{3,4}$ bestimmt. Die Testungen der reinen Neurohormone ergaben außer einer wesentlich höheren spezifischen Aktivität die mit den ungereinigten Produkten erreichten Wirkungen.



Abb. 1. Trennung an Sephadex G-25. a) Neurohormon D und Aminosäuren; b) Neurohormon C und Aminosäuren.

Neurohormon D: Steigerung der Herztätigkeit von Periplaneta mit Herzblock in der Diastole. Aktivierung der Nerventätigkeit von Periplaneta (gleiche Wirkung wie Vasopressin). Aufhellung von Leander und Crangon.
N e u r o horm on C: Steigerung der Herztätigkeit von Periplaneta mit Herzblock in der Systole. Hemmung der durch das Neurohormon D ausgelösten gesteigerten Nerventätigkeit von Periplaneta (gleiche Wirkung wie Oxytocin). Verdunkelung der Garnelen Leander und Crangon.

Eine Inaktivierung der Neurohormone wurde wie bei den enzymatischen Abbauversuchen ${ }^{2,3}$ durch Hydrolyse mit Salzsäure, Oxydation mit Perameisensäure, Entschwefelung mit aktivem Nickel und Desaminierung mit nitrosen Gasen erreicht.

Gleichlaufende Stoffwechseluntersuchungen mit ${ }^{35} \mathrm{~S}$ an Periplaneta ergaben für die Neurohormone eine autoradiographisch nachweisbare geringe Aktivität ${ }^{11}$.

Bis zur endgültigen Strukturaufklärung der Neurohormone C und D nach der Aufarbeitung größerer Mengen des biologischen Materials kann aus den bisherigen Ergebnissen folgendes geschlossen werden: Die Neurohormone sind Peptide mit schwefelhaltigen Aminosäuren im Molekül. Die Analogie der biologischen Wirkungen und des Verhaltens der Arthropodenwirkstoffe und der Wirbeltierhormone Oxytocin und Vasopressin führt zu der Vermutung einer der den Oktapeptiden gleichen oder ähnlichen Struktur der Neurohormone C und $\mathrm{D}$.

Herrn Dr. H. Unger, Magdeburg, danken wir recht herzlich für die Durchführung der elektrophysiologischen Versuche an Periplaneta.

9 H. UNGER, Biol. Zbl. 76, 204 [1957]; Naturwissenschaften 43, 66 [1956].

10 H. Unger u. W. Kalkoff, Acta biol. med. german. 13, 532 [1964] ; Zool. Jb. Physiol. 71, 727 [1965].

11 W. KAPITZA, unveröffentlicht.

\section{Zur Chemie chilenischer Flechten XI ${ }^{1}$}

\section{Über die Inhaltsstoffe von Ramalina tigrina FOLLM. und Ramalina inanis MONT.}

\author{
Siegfried Huneck \\ Institut für Pflanzenchemie der Technischen Universität \\ Dresden in Tharandt \\ und \\ Gerhard Follmann \\ Botanisches Museum, Berlin ${ }^{2}$ \\ (Z. Naturforschg. 21 b, 713-714 [1966] ; eingegangen am 9. April 1966)
}

Ramalina tigrina FOLLM., eine neue Ramaloidee aus der vorwiegend südamerikanischen Ceruchisgruppe, deren Diagnose a. a. $O$. veröffentlicht wird, stellt eine epiphytische (ausnahmsweise auch terricole), ärohygrophile Flechtenart der nordchilenischen Dornstrauchund Sukkulenten-Formationen dar. Vorläufig darf sie als Endemit des westlichen Andenabfalls zwischen

1 28. Mitt. über Flechteninhaltsstoffe. 27. Mitt.: S. Huneck u. J.-M. LeHN, Z. Naturforschg. 21 b, 299 [1966]. $18-25^{\circ} \mathrm{SB}$ angesprochen werden. Pflanzensoziologisch tendiert sie zum nebelanzeigenden Anaptychietum intricatae FOLLM. (Teloschistidion chrysophthalmi FOLLM. ${ }^{3}$ ).

Die Rinde der gelbgrünen, schwarzgestreiften Ramalina tigrina FOLLM. färbt sich mit Kalilauge plus Natriumhydrochlorit schwach gelb, während die untere Rindenschicht mit $p$-Phenylendiamin eine schwache Gelbfärbung zeigt. Die Apothecienscheiben färben sich mit Jodjodkalium-Lösung blauschwarz.

$73,0 \mathrm{~g}$ Flechte vom Cerro Moreno (600 m ü. M., Provinz Antofagasta) werden bei $90^{\circ}$ getrocknet, gemahlen und $12 \mathrm{Stdn}$. mit $100 \mathrm{ml}$ Äther farblos gewaschen und aus Methanol umkristallisiert: 1,55 $\mathrm{g}$ Nadeln vom Schmp. $216-217^{\circ}$, identisch mit (-)-16a-Hydroxykauran $(=$ Ceruchinol $=$ Ceruchdiol $)$. Aus dem mit Natriumhydrogencarbonat-Lösung und Natronlauge ausgeschüttelten ätherischen Filtrat lassen sich weitere 0,4 g Ceruchinol gewinnen; Gesamtausbeute: 1,95 g $(2,7 \%)$.

2 Anschrift: Botanisches Museum, 1 Berlin 33, Königin-LuiseStr. 6-8.

3 G. Follmann, Ber. dtsch. bot. Ges. 74, 495 [1961]. 